\title{
a.
}

EPL FOR THE IYL 2015

_ Giorgio Benedek ${ }^{+}$and Graeme Watt ${ }^{\star}$ - DOI: http://dx.doi.org/10.1051/epn/2016202

- 'EPL Editor in Chief; 'EPL Executive Editor

The European Physical Society has greatly contributed to the celebration of the International Year of Light and Light-Based Technologies (IYL 2015) in various ways [1]. In particular Europhysics News has concluded the year 2015 with a Special Issue on the Science of Light which illustrates, through six short reviews, the leading role that Europe in maintaining in several areas of the physics of light and its applications. EPL has played its part by publishing a series of invited IYL Perspectives.

\section{IYL Perspectives}

As announced in an EPL Editorial [2] Perspectives are a new type of invited letter-sized reviews on recent advances in some relevant area of physics, presented in a style accessible to a general physics readership. Fourteen IYL Perspectives have appeared so far, covering various important aspects of the physics of light. They have been intercalated by eight Perspectives in other areas of physics, though it is hard to imagine some area of physics that doesn't involve light in some way. The whole set of Perspectives is available in http://iopscience.iop.org/02955075/focus/Perspectives. The IYL Perspectives (IYL-PS) appeared in EPL are in many respects complementary to the six EPN reviews: together the set of twenty papers appearing in our two journals form a coherent set covering the most exciting aspects of the physics of light.

\section{The cosmic messenger}

Light, as a messenger from the remotest parts of the universe in time and space, tells us about its primordial constitution through the cosmic microwave background (CMB). Jo Dunkley in her IYL-PS [3] summarizes the recent $\mathrm{CMB}$ data collected by the Planck satellite and ground-based experiments, and the cosmological properties extracted from that rich information, or expected from upcoming observations. Cosmology relies on general relativity and its validity can now be tested with unprecedented accuracy by means of atomic clocks. The coherent optical link method, where ultra-stable laser light is transferred along optical fibres to synchronize remote clocks, was shown to attain frequency resolutions better than $10^{-18}$ in $1000 \mathrm{~s}$ over $1000 \mathrm{~km}$, thus permitting synchronization over a continental scale (Fig. 1). Calonico et al. discuss in Light and the Distribution of Time [4] the tremendous progress made in this field and the vast implications in metrology, geodesy, astronomy and cosmology, e.g., in the search for dark matter.

vFIG. 1: Light and the distribution of time: Optical Fiber Links in Europe. Solid Lines: existing optical links. Dashed line: ex tensions in progress (from Calonico, Inguscio and Levi [4]).

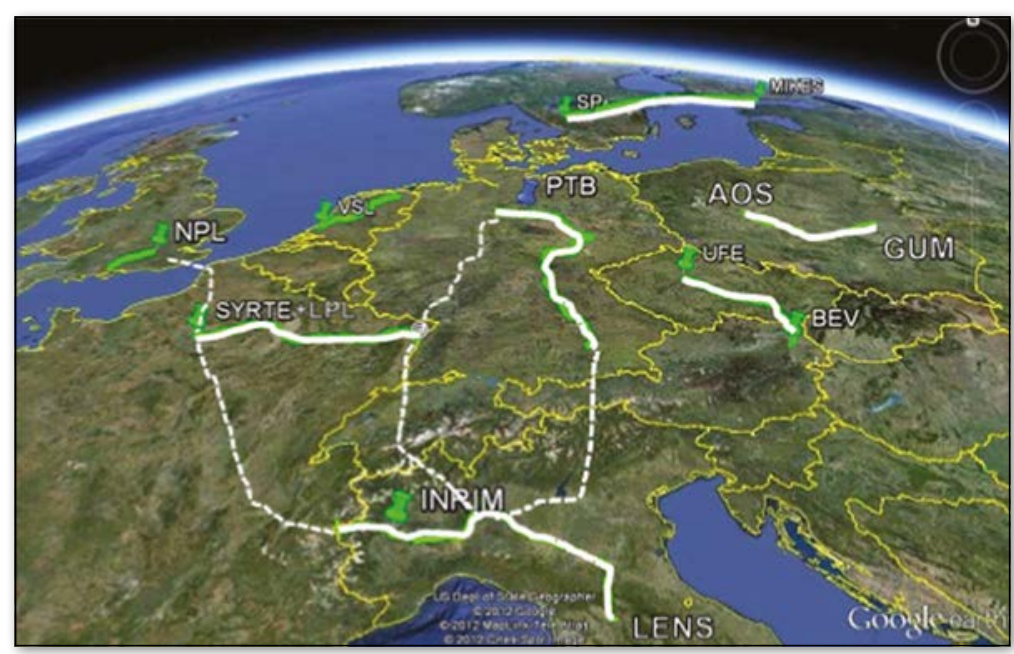




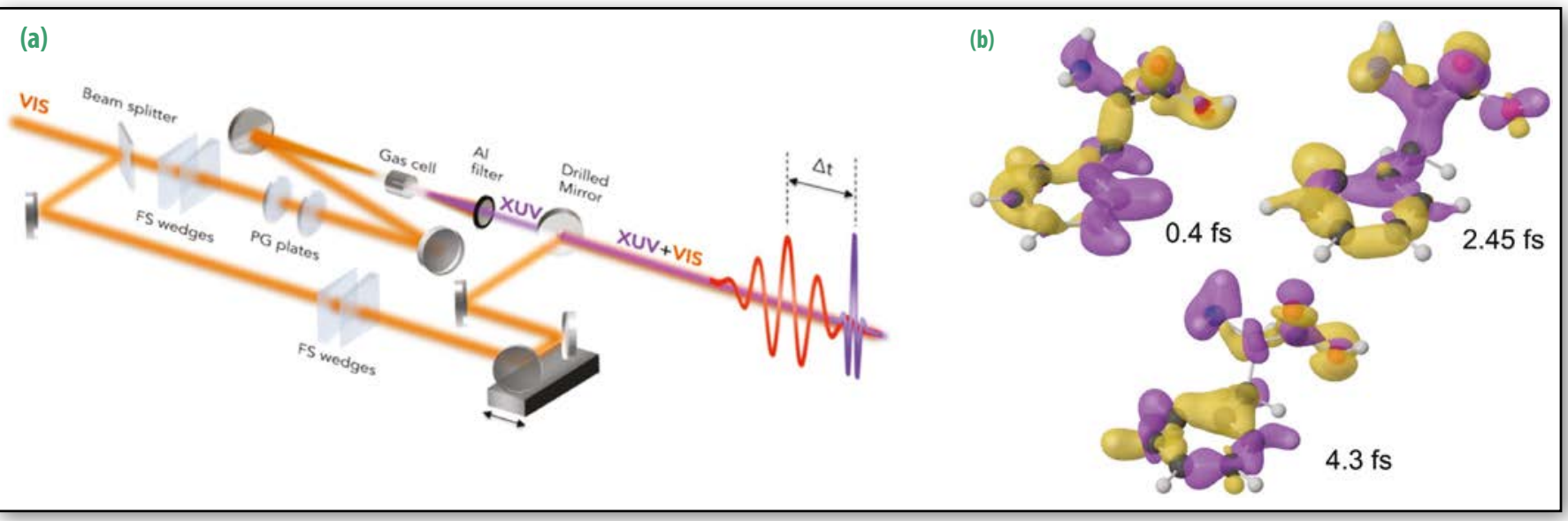

$\Delta$ FIG. 2: Light at the extreme: (a) Attosecond experimental setup. The FS wedges: ultrathin fused silica wedges for fine dispersión compensation; PG plates: birefringent plates for polarization gating; Al filter: $100 \mathrm{~nm}$ thick aluminum filter to block fundamental radiation and to compensate for the intrinsic chirp of attosecond pulses. (b) Snapshots of the calculated electron dynamics for three particular pump-probe delays. The figure shows the variation of the hole density with respect to its time-averaged value: yellow and purple correspond to positive and negative values, respectively (from Cerullo, De Silvestri and Nisoli [10])

\section{Extreme light}

Attempts to reproduce in ground-based experiments the extreme conditions of matter considered in astro-particle physics are so far the domain of large accelerators. The discovery of laser chirped-pulse amplification (CPA) made in 1985 by Strickland and Mourou [5] and subsequent great advancements [6-8] now allow production of giant laser wakefield accelerations in a solid, and high-energy radiation and particle beams with an extremely short time structure [8] : the CERN on a chip, as Gérard Mourou likes to say! The art of pulse compression down to the atto-second scale has received a strong boost from the Milan Polytechnic group [9,7]: now Cerullo, De Silvestri

\footnotetext{
>FIG. 3:

(a) Schematic

band diagram of a quantum-well tunnel-

junction transistor

laser (QW TJ-TL)

shown with a generic

resonator cavity;

(b) Microwave

signal mixing with

a common-emitter

tunnel junction

transistor laser

with a pair of input

sinusoidal signals:

one $\left(f_{1}=2.0 \mathrm{GHz}\right)$

at the base using

current modulation,

and the other

$\left(f_{2}=2.1 \mathrm{GHz}\right)$ at

the collector using voltage modulation.

The optical output

with harmonics

actually extends up

to the $11^{\text {th }}$ order,

$\left(4 f_{1}+7 f_{2}=22.7 \mathrm{GHz}\right)$

despite being

limited by amplifier

bandwidth (from [15]).
}

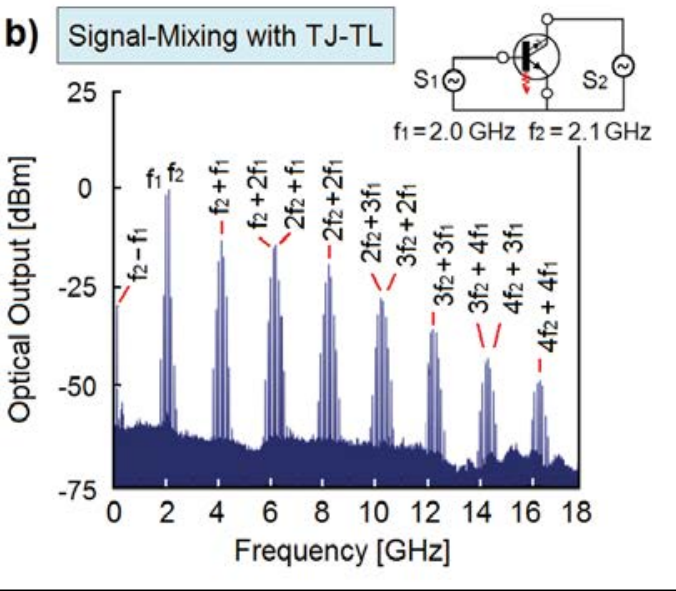

and Nisoli explain in their IYL-PS [10] how the motion of atoms and electrons can be followed in real time, thanks to these achievement, even across organic molecules (Fig. 2).

The subject of high-intensity laser-atom interactions has been reviewed by Charles Joachain, whose Perspective, starting with a nice historical introduction, has been chosen as a prelude to the IYL series in EPL and was published in the final volume of 2014 [11]. Large-scale European ultra-intense laser facilities like ELI, LMJ and Apollon will soon become operative. The study of condensed matter under extreme conditions planned at PETAL (Petawatt Aquitaine Laser), inaugurated last September at the CEA-CESTA Laser MegaJoule facility near Bordeaux [12], is being illustrated in a forthcoming IYL-PS by Dimitri Batani, anticipated however by an Editor's Choice EPL on shocked-compressed water [13]. There is great expectation from ultra-intense laser facilities for controlled inertial fusion: Stefano Atzeni reviews the state of the art in his IYL-PS [14], reporting on the recent breakthroughs and the potential of alternative schemes, in particular direct-drive shock ignition.

\section{From LED to nano-photonics}

A much more familiar use of light is for illumination. A major scientific revolution of the past century was the first realization in 1962 of a visible light-emitting diode (LED) by Nick Holonyak Jr. and S. F. Bevacqua: Nick Holonyak accepted to write with Milton Feng a Perspective for EPL - a great start for the 2015 IYL-PS series! It is well known, however, that Nick Holonyak and Milton Feng made another breakthrough in 2005, the creation of the transistor laser (Fig. 3): this is actually the subject chosen for their Perspective [15]. Most welcome, in view of the great promise that the transistor laser holds in the fields of electro-optics, such as for nonlinear signal mixing, frequency multiplication, negative 
feedback, optoelectronics logic gates and nanophotonics applications! The descent of optics and photonics to the nanoscale was actually celebrated by Zhi-Yuan Li (Beijing Academy of Sciences), who explored in his IYL-PS [16] the concepts, insights, methodologies, and technologies in nanophotonics setting a solid platform to achieve better future technologies that use light as a carrier of energy and information, and as a medium to probe and manipulate the intrinsic properties of matter via light-matter interaction. The IYL-PS by the Madrid group [17] and one by Tongtong Zhu and Rachel Oliver at Cambridge [18] envisage the new avenues that single photon sources implemented in nitride nano-structures will open in quantum photonics and information processing.

\section{Quantum electro-dynamics}

Cavity Quantum Electrodynamics (CQED), where a single two-level atom is coupled to a few photons stored in a single mode of a high-quality resonator, besides its fundamental interest in the study of coherent atom-field interactions, provides an ideal situation for the realization of quantum information protocols. In their IYL-PS on trapped quantum light [19], Michel Brune and Jean-Michel Raimond illustrate the fundamental interest of CQED in connection with recent experiments, performed with circular Rydberg atoms and superconducting millimetre-wave cavities. Fundamental aspects of QED can be tested in Casimir force experiments, e.g., by measuring the Casimir torque induced by quantum vacuum fluctuations between two nanostructured plates (Fig. 4), as illustrated in the IYL-PS by Guérout et al. [20], or with the experiment proposed by D. S. Ether Jr. et al. [21], where optical tweezers are used to probe the Casimir interaction between microspheres inside a liquid medium.

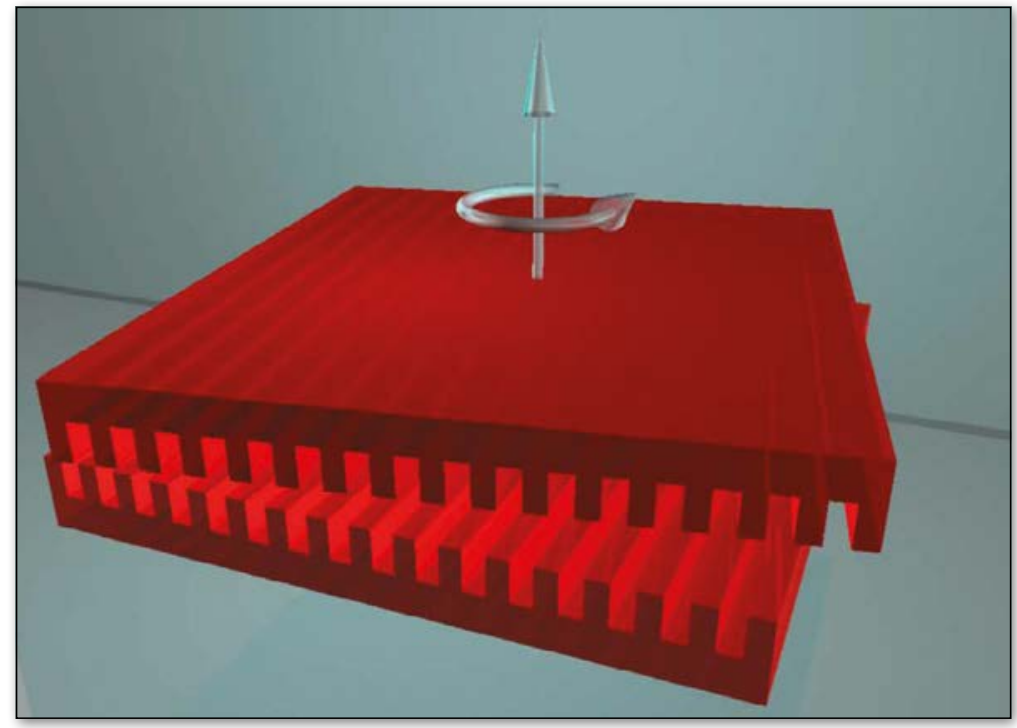

\ FIG. 4: Casimir torque between nanostructured plates: Quantum vacuum fluctuations produce Casimir forces when scattered by external boundaries. The figure shows a sketch of the configuration giving rise to a Casimir torque (from Guérout, Genet, Lambrecht and Reynaud [20]).

\section{Light for graphene and nano-medicine}

New domains of optics are worth being emphasized. One is the use of ultrafast $\mathrm{THz}$ spectroscopy for the characterization of ultrafast charge carrier dynamics as, e.g., that of Dirac electrons and holes in graphene. The recent experimental work in this rapidly growing and exciting area is reviewed in the IYL-PS by Ivan Ivanov et al. at the MPI for Polymer Research in Mainz [22]. The other domain is optics in the vast and rapidly growing field of nano-medicine, a field which would deserve an entire series of IYL Perspectives. Just one fascinating example, due to Vincent Daria and Hans Bachor [23], has been chosen, where optics is shown to be a powerful tool for the analysis of neuronal information processing. In parallel to imaging, laser-based stimulation, e.g., of light-sensitive actuators and reporters in neuronal activity, has become

V FIG. 5: Using light to probe neuronal function: (a) Photochemical reaction for glutamate uncaging. (b) Illustration of $2 \mathrm{P}$ uncaging near dendritic spines where neurotransmitter-gated ion channels are located. (c) Light-activated ion channels (e.g., channelrhodopsin) expressed in cell membranes. Ion channels are not drawn to scale (from Daria and Bachor [23]).

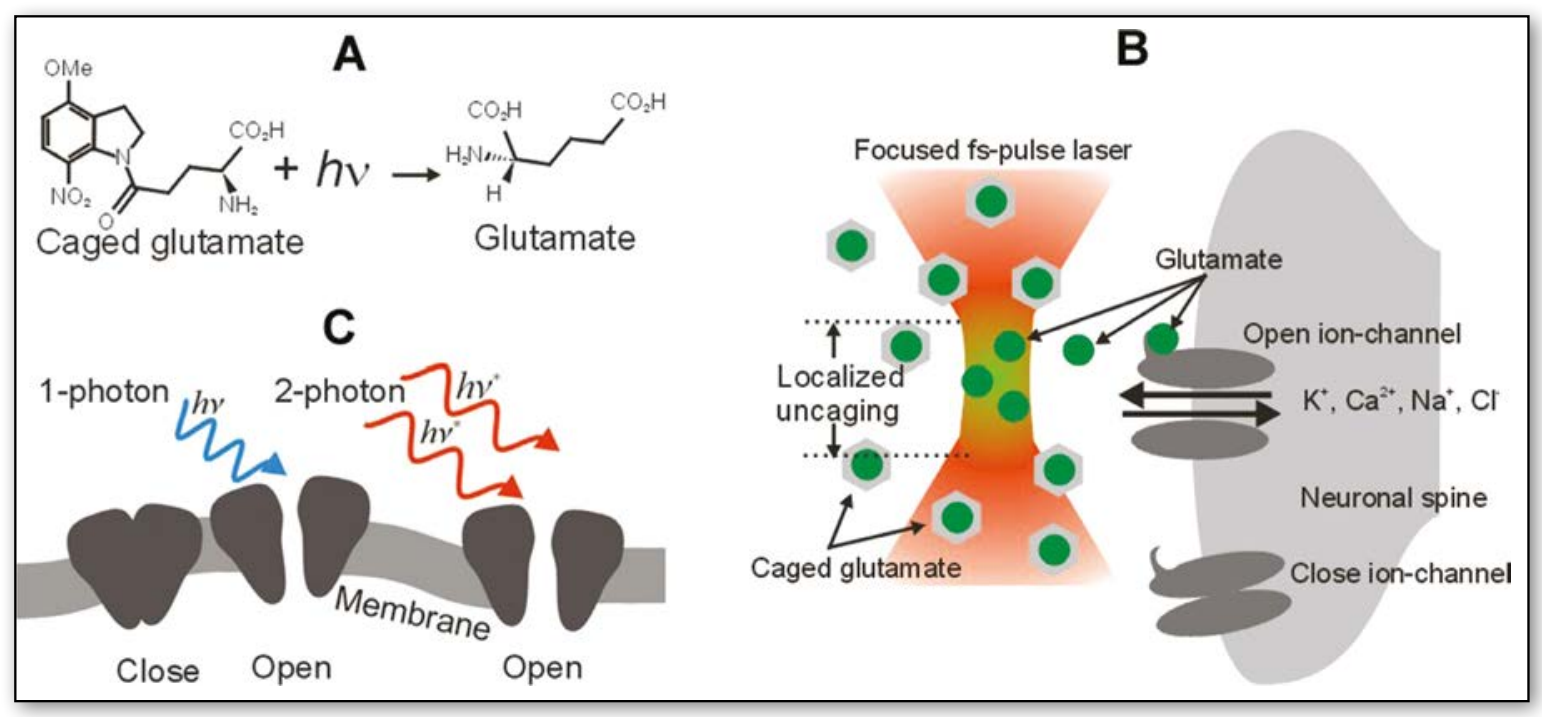


a well-established method to investigate neuronal activity (Fig. 5). Even in in vivo experiments the analysis is reaching a resolution to the level of single-neuron response. The authors argue that, besides providing fundamental information on the brain processes and development, optical methods may have medical applications, e.g., in the analysis of mental disorders.

\section{Ultra-cold atoms and the unity of physics}

In our descent from the highest energies of astroparticle physics and cosmology, down to the lower and lower energies through ordinary condensed matter, we finally reach the world of ultra-cold atoms. This is another

v FIG. 6: Cold atoms: A field enabled by light: (a) A laser cooling experiment. Inside the vacuum chamber a ball of $\sim 10^{9}$ cold 6 Li atoms (the white spot in the centre), at a temperature of $\sim 1 \mathrm{mK}$, glows while scatters photons from the beams of a magneto-optical trap (courtesy of G. Roati, LENS). (a,b) Laser-cooled atomic clocks: (a) In state-of-the-art microwave clocks an atomic fountain provides long interrogation times on the order of one second (a). In the new generation of optical clocks the atoms are trapped in an optical lattice, which freezes the atomic motion and provides Doppler- and recoil-free measurements (b) (from Fallani and Katsberg [24]).

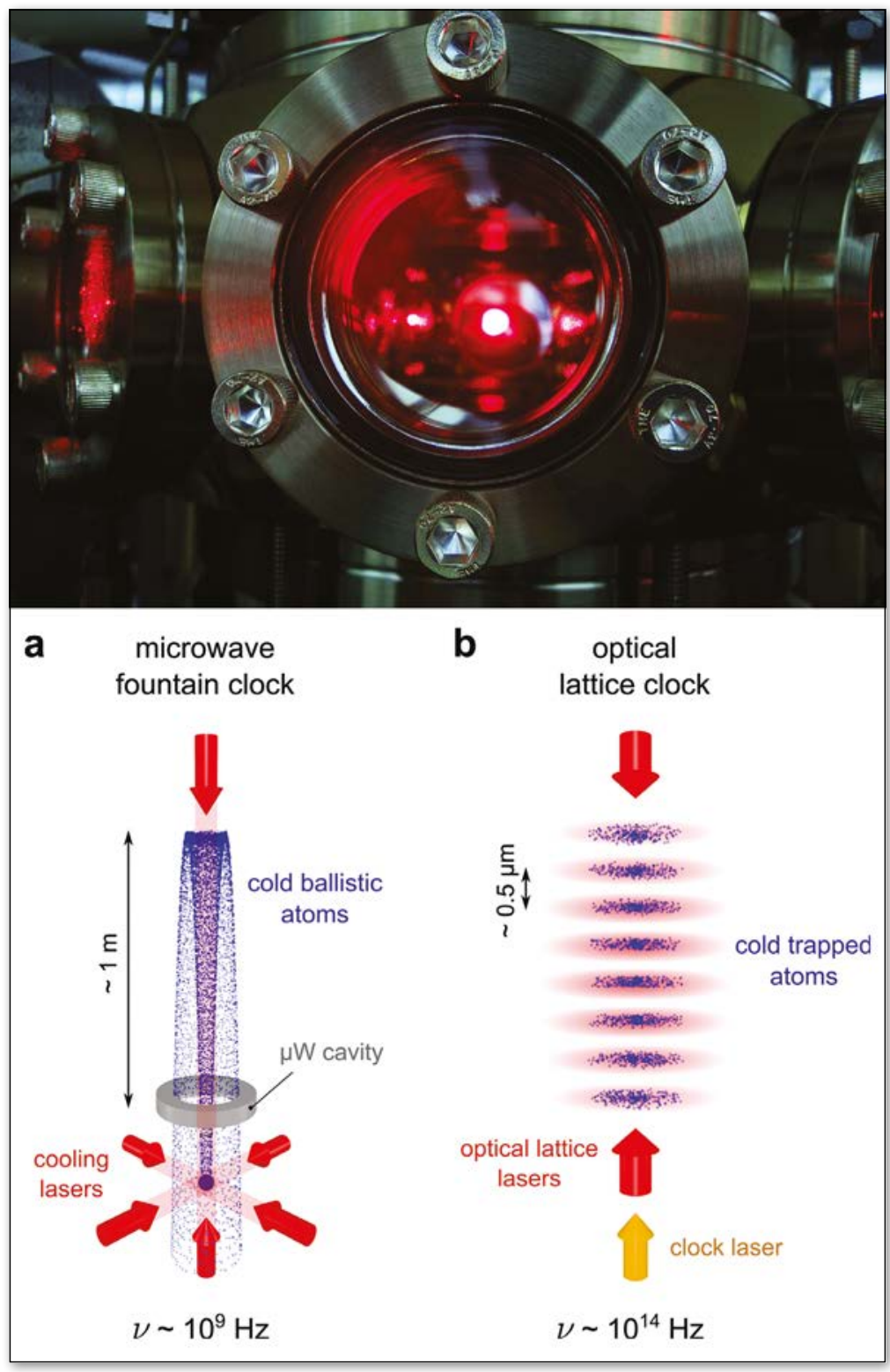

exciting area of modern physics enabled by light via laser cooling and the realization of optical traps and lattices for quantum gases. The thirty-year history of laser cooling up to the recent achievements is summarized in the IYLPS by Fallani and Kastberg [24]: the demonstration of Bose-Einstein condensation, the simulation and testing of fundamental quantum properties, the realization of laser-cooled clocks for high precision measurements (Fig. 6), the construction of customized synthetic potentials to simulate interactions in other domains of physics, e.g., in QCD, are just examples of the new world of ultra-low temperatures made accessible by light. Much of this field connects with the areas of physics first mentioned in this summary, astroparticle physics, general relativity, cosmology, and fundamental metrology. The circle closes, to remind the essential unity of physics: a unity which goes well beyond the instrumental aspects, to acquire a high conceptual value. For example, the concept of perfect fluid can apply to a strongly interacting ultra-cold Fermi gas as well as to the quark-gluon plasma, or in cosmology to describe Friedmann-Lemaître-Robertson-Walker universe evolution.

\section{Perspectives for a stronger EPL}

Perspectives have met a great success in terms of downloaded content: they systematically appear among the top-10 most-read EPL letters. There seems to be a need for short reviews intended for an audience of physicists working in adjacent fields who want something more technical and richer in references than the usual physics magazine article, but shorter and easier to read than a standard review article written for specialists. Perspectives constitute a further well-received service that the physical societies owning EPL, offer to the worldwide community of physicists. Thus the Perspective series will continue, possibly with a substantial extension in number. However, invited Perspectives are only a small fraction of EPL letters. Most published EPL letters are selected (a bit more than $1 / 3$ ) out of the submitted manuscripts by an Editorial Board of about 70 outstanding colleagues who represent all branches of physics and all continents, and are entirely and uniquely responsible of the peer-review process.

The number of excellent physics letters produced world-wide largely exceeds what high-impact letter journals, either general or specialized, can host. Not necessarily the best papers are published in the highest IF journals, since the scientific prestige of the paper mostly depends on the scientific prestige of the editors more than on the visibility of the journal. If you feel that your recent work either changes a paradigm; or opens a novel significant research area in physics; or presents a substantial progress in a relevant field of physics; or makes a valuable contribution in a field of broad general interest, closely related to physics, do not hesitate and submit to EPL! 


\section{About the Authors}

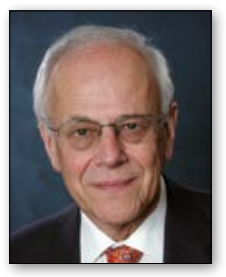

Giorgio Benedek, former Professor of Structure of Matter at the University of Milano-Bicocca and Visiting Professor at the Donostia International Physics Center in San Sebastian, is presently the EPL Editor-in-Chief and Fellow of EPS. He is a condensed matter physicist mainly interested in surface dynamics, surface phonon spectroscopy, electron-phonon interaction, and low-dimensional systems such as nanostructured carbon and quantum droplets.

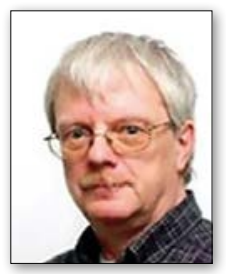

Graeme Watt has been the Executive Editor for EPL since 2007. In addition to overseeing the smooth efficient publication of the journal he is also responsible for strategy and development. He can be located promoting EPL at many conferences.

\section{References}

[1] J. M. Dudley, Phys. Rev. Lett. 114, 120001 (2015)

[2] G. Benedek and G. Watt, EPL 107, 40000 (2014)

[3] J. Dunkley, EPL 111, 49001 (2015)

[4] D. Calonico, M. Inguscio and F. Levi, EPL 110, 40001 (2015)

[5] D. Strickland and G. Mourou, Optics Comm. 56, 219 (1985)

[6] G.A. Mourou, T. Tajima and S.V. Bulanov, Rev. Mod. Phys. 78, 309 (2006).

[7] O. Svelto, Principles of Lasers, $5^{\text {th }}$ edn, Springer, New York (2010).

[8] G. Mourou, J. A. Wheeler, and T. Tajima, Europhysics News 46/5\&6, 31 (2015)

[9] M. Nisoli, et al., Appl. Phys. B: Lasers and Optics 65, 189 (1997).

[10] G. Cerullo, S. De Silvestri and M. Nisoli, EPL 112, 24001 (2015)

[11] C. J. Joachain, EPL 108, 44001 (2014)

[12] L. Bergé, e-EPS, 22 Oct (2015)

[13] D. Batani, EPL (to appear, 2016); see also D. Batani , et al., EPL 112, 36001 (2015)

[14] S. Atzeni, EPL 109, 45001 (2015)

[15] M. Feng and N. Holonyak jr., EPL 109, 18001 (2015)

[16] Z.-Y. Li, EPL 110, 14001 (2015)

[17] E. Chernysheva, et al., EPL 111, 24001 (2015)

[18] T. Zhu and R. A. Oliver, EPL 113, 38001 (2016)

[19] M. Brune and J. M. Raimond, EPL 110, 20001 (2015)

[20] R. Guérout, C. Genet, A. Lambrecht and S. Reynaud, EPL 111, 44001 (2015)

[21] D. S. Ether jr., et al., EPL 112, 44001 (2015)

[22] I. Ivanov, M. Bonn, Z. Mics and D. Turchinovich, EPL 111, 67001 (2015)

[23] V. R. Daria and H.-A. Bachor, EPL 111, 38001 (2015)

[24] L. Fallani and A. Kastberg, EPL 110, 53001 (2015)

\section{[ Letter to the Editor $]$}

by Adelbert Goede

Fellow of the European Physical Society

DOI: http://dx.doi.org/10.1051/epn/2016203

\section{The need for Basic Energy Research}

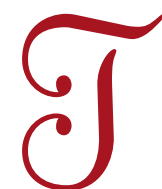

he Paris Conference of the United Nations Framework Convention on Climate Change (UNFCC) may have brought relief to some, the fact remains that climate models, the basis of the politically negotiated limit on earth surface temperature rise, are far from perfect. The ocean exerts large inertia on the coupled atmosphere-ocean system and its interaction is less well understood. For example,Atlantic multi-decadal temperature oscillation, the global-warming hiatus, was not captured by these models. Meridional overturning time scales of the ocean are expressed in centennials rather than decades, which stretches the predictive power of models. Models are starved of deep sea data which carry the heat; below 700m depth hardly any measurement exist to validate the models. This calls for better models and more measurements, lest public opinion turns against current climate policy measures for lack of credibility of the climate forecast.

This is hardly EPS expertise and not the point anyway. At this point in time, given the uncertainties, it is good policy to work on solutions, rather than argue about (imperfect) climate model predictions. The energy transition is EPS core business and this is what we should be concentrating on.

The energy transition is increasingly hampered by its focus on the energy source rather than the system as a whole. For example, large scale deployment of wind farms is based on the upscaling of existing wind turbine technology, rather than basic research needed to solve the intermittency problem at system level including energy storage. Public money is spent on deployment of today's technology. As a consequence, perverse feed-in tariffs, destructive cross border transport and wasteful overcapacity characterise this corner of the renewable energy market.

The EU has set ambitious energy and climate targets. The Strategic Energy Technology (SET) Plan shows what research is needed. Sadly, when it comes to implementation, the EU Horizon 2020 Energy Work Programme 2016-2017 offers precious little opportunity in basic energy research. Most calls are directed at high Technological Readiness Level, aimed at implementation of today's technology. The 2030-2050 energy transition, however, requires investment in basic research and innovation, enabling tomorrow's technology. The EPS should stand up against current EU implementation practise and advocate the need for basic energy research both in Brussels and with their Member State masters. 